\title{
Determination of the local dark matter density in our Galaxy
}

\author{
M. Weber and W. de Boer
}

\begin{abstract}
Institut für Experimentelle Kernphysik, Karlsruher Insitut für Technologie (KIT), PO Box 6980, 76128 Karlsruhe, Germany
e-mail: Markus.Weber@ekp.uni-karlsruhe.de
\end{abstract}

Received 30 September 2009 / Accepted 9 October 2009

\section{ABSTRACT}

\begin{abstract}
Context. The rotation curve, the total mass and the gravitational potential of the Galaxy are sensitive measurements of the dark matter halo profile.

Aims. Cuspy and cored DM halo profiles are analysed with respect to recent astronomical constraints in order to constrain the shape of the Galactic DM halo and the local DM density.

Methods. All Galactic density components (luminous matter and DM) are parametrized. Then the total density distribution is constrained by astronomical observations: 1) the total mass of the Galaxy, 2) the total matter density at the position of the Sun, 3) the surface density of the visible matter, 4) the surface density of the total matter in the vicinity of the Sun, 5) the rotation speed of the Sun and 6) the shape of the velocity distribution within and above the Galactic disc. The mass model of the Galaxy is mainly constrained by the local matter density (Oort limit), the rotation speed of the Sun and the total mass of the Galaxy from tracer stars in the halo. Results. We showed from a statistical $\chi^{2}$ fit to all data that the local DM density is strongly positively (negatively) correlated with the scale length of the DM halo (baryonic disc). Since these scale lengths are poorly constrained the local DM density can vary from 0.2 to $0.4 \mathrm{GeV} \mathrm{cm}^{-3}\left(0.005-0.01 M_{\odot} \mathrm{pc}^{-3}\right)$ for a spherical DM halo profile and allowing total Galaxy masses up to $2 \times 10^{12} M_{\odot}$. For oblate DM haloes and dark matter discs, as predicted in recent $N$-body simulations, the local DM density can be increased significantly.
\end{abstract}

Key words. Galaxy: halo - Galaxy: structure - Galaxy: kinematics and dynamics - Galaxy: fundamental parameters Galaxy: general

\section{Introduction}

The best evidence for dark matter (DM) in galaxies is usually provided by rotation curves, which do not fall off fast enough at large distances from the centre. This can be understood, either by assuming Newton's law of gravitation is not valid at large distances, the so-called MOND (modified newtonian dynamics) theory (Bekenstein \& Milgrom 1984; Bienayme et al. 2009), or the visible mass distribution is augmented by invisible mass, i.e. DM. For a flat rotation curve the DM density has to fall off like $1 / r^{2}$ at large distances.

Given the overwhelming evidence for DM on all scales from the flatness of the universe combined with gravitational lensing and structure formation we assume DM exists and try to constrain the DM density from dynamical constraints, for which better data became available in recent years:

1. the total mass of the Galaxy has to be about $10^{12}$ solar masses (Wilkinson \& Evans 1999; Battaglia et al. 2005; Xue et al. 2008);

2. the total mass inside the solar orbit is constrained by the well-known rotation speed of the solar system;

3. the total matter density at the position of the Sun from the gravitational potential determined from the movements of local stars as measured with the Hipparcos satellite (Holmberg \& Flynn 2004);

4. the surface density of the visible matter at the position of the Sun (Naab \& Ostriker 2006, and references therein);

5. the surface density of the total matter at the position of the Sun (Kuijken \& Gilmore 1991; Holmberg \& Flynn 2004; Bienayme et al. 2005);
6. the shape of the rotation curve within Galactic disc (Sofue et al. 2008, and references therein);

7. the velocity distribution above the Galactic disc $(z>4 \mathrm{kpc})$ (Xue et al. 2008).

Towards the Galactic centre (GC) $N$-body simulations of structure formation predict a DM density, which diverges at the centre, thus developing a so-called cusp, with the slope varying more like $1 / r$ than $1 / r^{2}$ (Navarro et al. 1997; Moore et al. 1999; Ricotti 2003). On the contrary, rotation curves of nearby dwarf galaxies do not show such a cusp, but rather a constant density near the centre, a so-called core (Oh et al. 2008; Gentile et al. 2007; Salucci et al. 2007). $N$-body simulations including the complicated baryonic disc formation indicated that the cusps may have been destroyed by the fluctuation in the gravitational potential of the baryons, which is much stronger than the potential caused by the DM in the centre (Mashchenko et al. 2006). In addition, the DM may form a disc like structure by the infall of DM dwarf galaxies, as shown by recent high resolution $N$-body simulations (Purcell et al. 2009). Evidence for substructure of DM inside the disc is supported by the structure in the gas flaring (Kalberla et al. 2007), by the peculiar change of slope for the rotation curve inside the disc, which is not observed for stars outside the disc (Sect. 3.2) and by the structure in the diffuse gamma radiation (de Boer et al. 2005).

Such substructure will not be investigated in this paper, but smooth DM haloes with different (cored and cuspy) profiles will be compared with all available data. These will result in lower limits on the local DM density, since dark matter discs or other local substructure will only enhance the local density.

A reliable determination of the local DM density is of great interest for direct DM search experiments, where elastic 
collisions between WIMPs and the target material of the detector are searched for. This signal is proportional to the local density. A review on direct searches can be found in the paper by Spooner (2007).

The structure of the paper is as follows: in Sect. 2 the parametrization of the luminous matter and five different DM halo profiles are given. In Sect. 3 the experimental data used to determine the mass model are discussed. Then the numerical determination of the mass model parameters using a $\chi^{2}$ fit of all dynamical constraints is discussed in Sect. 4. At the end a summary of the results is given.

\section{Parametrization of the density distributions}

In order to constrain the mass model of the Galaxy by data it is convenient to have a parametrization for both, the visible an dark matter density. These parametrizations are introduced here.

\subsection{Parametrization of the luminous matter density}

The density distribution of the luminous matter of a spiral galaxy is split into two parts, the Galactic disc and the Galactic bulge. The parametrization of the density distribution of the bulge is adapted from the publication by Cardone \& Sereno (2005)

$$
\begin{aligned}
\rho_{\mathrm{b}}(r, z) & =\rho_{\mathrm{b}} \cdot\left(\frac{m}{r_{0, \mathrm{~b}}}\right)^{-\gamma_{\mathrm{b}}} \cdot\left(1+\frac{\tilde{r}}{r_{0, \mathrm{~b}}}\right)^{\gamma_{\mathrm{b}}-\beta_{\mathrm{b}}} \exp \left(-\frac{\tilde{r}^{2}}{r_{\mathrm{t}}^{2}}\right), \\
\tilde{r}^{2} & =\sqrt{x^{2}+y^{2}+\left(z / q_{\mathrm{b}}\right)^{2}} .
\end{aligned}
$$

For a good description of the RC near the GC the parameters of the bulge profile are found to be $\gamma_{\mathrm{b}}=\beta_{\mathrm{b}}=1.6, q_{\mathrm{b}}=0.61$, $r_{\mathrm{t}}=0.6 \mathrm{kpc}, r_{0, \mathrm{~b}}=0.7 \mathrm{kpc}$ and $\rho_{\mathrm{b}}=360 \mathrm{GeV} \mathrm{cm}^{-3}$, which corresponds to approximately $9.5 M_{\odot} \mathrm{pc}^{-3}$. This high density might be influenced by the presence of black holes. At least one black hole with a mass of about $4.0 \times 10^{6} M_{\odot}$ has been observed in the GC by Reid (2009).

The stellar contribution of the Galactic disc is split into two discs - a thin and a thick disc - which are usually parametrized by an exponentially decreasing density distribution.

The parametrization of the Galactic disc is taken from the publication by Sparke (2007)

$\rho_{\mathrm{d}}(r, z)=\rho_{\mathrm{d}} \cdot \exp \left(-r / r_{\mathrm{d}}\right) \cdot \exp \left(-z / z_{\mathrm{d}}\right)$.

The parameter $\rho_{\mathrm{d}}$ describes the density of the Galactic disc at the GC while $r_{\mathrm{d}}$ and $z_{\mathrm{d}}$ describe the scale parameter in radial and vertical direction.

There is some freedom in the choice of the parameters for the Galactic disc. Its density in the GC is, as in case of the bulge, unknown, so it has to be a free parameter. For the scale radius we adopt the value from Hammer et al. (2007)

$r_{\mathrm{d}}=2.3 \pm 0.6 \mathrm{kpc}$.

The scale height $z_{\mathrm{d}}$ varies for the different components: young stars are born near $z=0$, so they have a much smaller scale height, while the older stars have been kicked around and reach scale heights up to $700 \mathrm{pc}$. The disc has two distinct populations with two different scale heights. The thin disc consists of young, bright and metal-rich stars which provide about $98 \%$ of the total stellar population (Gilmore \& Reid 1983). Its scale height was determined from star counts of stars with an absolute magnitude of $M_{V}>6$ to be $z_{\mathrm{d}} \approx 270 \mathrm{pc}$ (Kroupa et al. 1993). From measurements of the spatial distribution of $\mathrm{K}$ dwarf stars the vertical scale height turns out to be smaller than 250 pc (Robin et al. 1996). In the publication by Ojha et al. (1996) the scale height of the thin disc was determined for stars with $M_{V} \gtrsim 3.5$ to be $260 \pm 50$ pc. Furthermore the interstellar gas and dust contribution is also located in the thin disc. The thick disc consists of old, metal-poor stars and could be the result of either an earlier thin disc or the merging of a satellite galaxy with the MW (Robin et al. 1996). Its scale height lies between 700 and $1500 \mathrm{pc}$ and its local density is about $5 \%$ of the local stellar density of the thin disc. For that reason in this analysis the luminous matter is given only by the thin disc while small density contribution of the thick disc is neglected. For gas, which makes up $10 \%$ of the mass of the disc, the scale height varies with Galactocentric radius because of the decreasing gravitational potential at larger radii. Fortunately, the mass model of the Galaxy is not very sensitive to the exact value of the scale height, so it is fixed at $320 \mathrm{pc}$, which is close to the value adopted by Freudenreich (1998). The mass of the bulge was taken into account, but the detailed structure of the mass distribution (hole in the centre of the disc (Freudenreich 1998), the bar structure and the black hole) are not taken into account in the parametrization, since the parameters of interest, i.e. the DM halo parameters, are insensitive to the detailed mass distribution in the centre of the Galaxy, since the gravitational potential in the centre is completely dominated by the baryonic matter.

The parametrization of the visible mass discussed above leads to a mass of the Galactic bulge of about $1.1 \times 10^{10} M_{\odot}$. The mass of the Galactic disc varies for different fits because of the variation of the parameters $\rho_{\mathrm{d}}$ and $r_{\mathrm{d}}$. It is in the range of $5 \times 10^{10}$ to $7 \times 10^{10}$ solar masses. In addition to the luminous matter the density profile of the DM halo has to be parametrized. This is discussed in the following section.

\subsection{Parameterization of the dark matter density}

The first analytical analysis of structure formation in the Universe by Gunn (1977) predicted that DM in Galactic haloes are distributed according to a simple power law distribution $\rho(r) \propto r^{-\gamma}$. However, later studies based on numerical $N$-body simulations (Navarro et al. 1997; Moore et al. 1999) found that the slope of the density distribution in the DM halo is different for different distances from the GC. Today it is commonly believed that the profile of a DM halo can be well fitted by the universal function

$$
\begin{aligned}
\rho_{\chi}(r) & =\rho_{\odot, \mathrm{DM}} \cdot\left(\frac{\tilde{r}}{r_{\odot}}\right)^{-\gamma} \cdot\left[\frac{1+\left(\frac{\tilde{r}}{a}\right)^{\alpha}}{1+\left(\frac{r_{\odot}}{a}\right)^{\alpha}}\right]^{\frac{\gamma-\beta}{\alpha}}, \\
\tilde{r} & =\sqrt{x^{2}+\frac{y^{2}}{\epsilon_{x y}^{2}}+\frac{z^{2}}{\epsilon_{z}^{2}}} .
\end{aligned}
$$

Here, $a$ is the scale radius of the density profile, which determines at what distance from the centre the slope of the profile changes, $\epsilon_{x y}$ and $\epsilon_{z}$ are the eccentricities of the DM halo within and perpendicular to the Galactic plane and $r_{\odot}$ is the Galactocentric distance of the solar system. In the rest of this paper we will assume axisymmetrical haloes, i.e. $\epsilon_{x y}=1$, but $\epsilon_{z}$ is allowed to have values below or above 1, thus allowing oblate and prolate haloes. The parameter $\rho_{\odot, \mathrm{DM}}$ is the DM density of the halo at the position of the Sun. The parameters $\alpha, \beta$ and $\gamma$ characterize the radial dependence of the density distribution. For $r \approx a$ the slope of the halo profile is about $r^{-\alpha}$, for $r \gg a$ $\approx r^{-\beta}$ and for $r \gg a \approx r^{-\gamma}$. Different sets for these parameters 
Table 1. Parameter settings for the different DM halo profiles considered in this analysis.

\begin{tabular}{cccccc}
\hline \hline Profile & $\alpha$ & $\beta$ & $\gamma$ & $a[\mathrm{kpc}]$ & Reference \\
\hline NFW & 1.0 & 3.0 & 1.0 & 20.0 & Navarro et al. (1997) \\
BE & 1.0 & 3.0 & 0.3 & 10.2 & Binney \& Evans (2001) \\
Moore & 1.5 & 3.0 & 1.5 & 30.0 & Moore et al. (1999) \\
PISO & 2.0 & 2.0 & 0.0 & 5.0 & de Boer et al. (2005) \\
240 & 2.0 & 4.0 & 0.0 & 4.0 & \\
\hline
\end{tabular}

The parameters of the NFW profile are adapted from the publication by Navarro et al. (1997) but in this analysis a larger scale radius a is used.

were suggested by different analyses. Generally the results from numerical simulations show that the DM density in the GC is divergent. Such profiles are called "cuspy" profiles due to the cusp in the centre. In this analysis three cuspy profiles are considered. In the publication by Navarro et al. (1997) it was found that their simulation results can be approximated by a profile with a slope of $\gamma=1$ in the GC, a stronger decreasing slope of $\beta=3$ for large radii and $\alpha=3$ for the intermediate distances. This profile will be referred to as NFW. From later simulations by Moore et al. (1999) a profile with a somewhat steeper slope of $\gamma=1.5$ in the GC (hereafter Moore) was favoured. A third cuspy halo profile was introduced in (Binney \& Evans 2001). They found that the microlensing data towards the centre of the MW produced by the MACHO collaboration is compatible with a density profile with $\gamma \gtrsim 0.3$ (hereafter BE). Also recent $N$-body simulations showed a less cuspy profile for the clumpy component of the DM, which is usually called the Einasto profile (Ludlow et al. 2009). The amount of matter in clumps is uncertain, but usually considered small compared with the diffuse component (Springel et al. 2008a,b). By considering cusps with slopes between 0.3 and 1.5 one probably covers the range of possible slopes for cuspy profiles.

In contrast to cuspy profiles the density distributions preferred by observations of rotation curves of low surface brightness galaxies and dwarf spiral galaxies have a nearly constant DM density in the GC $(\gamma \approx 0)$ (Oh et al. 2008; Gentile et al. 2007; Salucci et al. 2007). Such profiles are called "cored" profiles due to the constant density in the kpc scale in the central region. Two different cored halo profiles are considered in this analysis. The first profile is called pseudo-isothermal profile (hereafter PISO) since it is an isothermal profile $\left(\propto r^{-2}\right)$ which is flattened in the centre. The second cored halo profile (hereafter 240) is similar to the PISO profile but decreases faster for large radii. The parameter settings of the several density profiles are shown in Table 1 . The local DM density $\rho_{\odot, \mathrm{DM}}$ is a priori an unknown parameter and therefore a degree of freedom in the density model. In the publications by Amsler et al. (2008); Gates et al. (1995) its value is quoted to be in the range 0.2$0.7 \mathrm{GeV} \mathrm{cm}^{-3}\left(0.005-0.018 M_{\odot} \mathrm{pc}^{-3}\right)$. In this analysis $\rho_{\odot, \mathrm{DM}}$ is left free. In Fig. 1 the different profiles are shown for equal masses within the solar orbit.

\section{Dynamical constraints on the mass model of the Milky Way}

As mentioned in the introduction the mass model of the Galaxy is largely constrained by the total mass of the Galaxy, the rotation velocity of the Sun at its orbital radius $r_{\odot}$ and the local matter density $\rho_{\odot \text {,tot }}=\rho_{\text {tot }}\left(r_{\odot}\right)$, known as the Oort limit. An additional constraint comes from the local surface density. The experimental input for these constraints is discussed first.

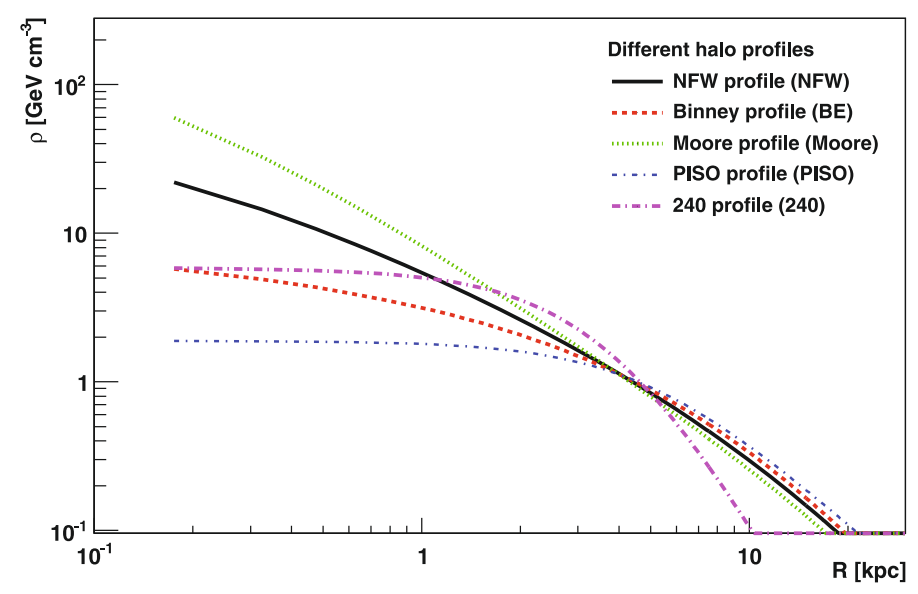

Fig. 1. Radial DM density distribution for the parameters given in Table 1 . The normalization $\rho_{\odot, \mathrm{DM}}$ of the halo profiles is fixed for each profile by the requirement of the rotation speed of the solar system.

\subsection{Total mass}

The total mass of the MW is an important quantity in order to constrain the DM density distribution. In general, it is measured indirectly either via the kinematics of distant halo tracer stars or satellite galaxies or the vertical scale height of the gas distribution of the Galactic disc, which can be measured at large distances from the GC.

The definition of the total mass of the Galaxy is difficult since a slowly decreasing density has an infinite extension. The total mass of a galaxy is conventionally defined as the mass within the so-called virial radius. At this radius the total mass of the accumulated density of the MW is equal to the mass of a homogeneous sphere with the constant density of 200 times the critical density of the Universe.

In the paper by Wilkinson \& Evans (1999) the mass of the MW was estimated from measurements of the radial velocities of 27 globular clusters and satellite galaxies for Galactocentric distances $R>20 \mathrm{kpc}$, using a Bayesian likelihood method and a spherical halo mass model with a truncated radius. They found a mass of the Galaxy within $50 \mathrm{kpc}$ of $M(50 \mathrm{kpc})=$ $5.4_{-3.6}^{+0.2} \times 10^{11} M_{\odot}$ and a total mass of $M_{\text {tot }}=1.9_{-1.7}^{+3.6} \times 10^{12} M_{\odot}$. A similar analysis was done with more tracer stars by Sakamoto et al. (2003); they find $M_{\text {tot }}=2.5_{-1.0}^{+0.5} \times 10^{12} M_{\odot}$. These measurements used a simple parametrization of the potential. Analyses using an NFW profile for the DM distribution usually find a lower total mass, given it steeper fall-off of the density profile at large distances.

Using a large sample of 2400 blue horizontal-branch (BHB) tracer stars from the Sloan Digital Sky Survey (SDSS) in the halo $(z>4 \mathrm{kpc}, R<60 \mathrm{kpc})$ and comparing the results with $N$-body simulations using an NFW profile Xue et al. (2008) find

$M_{R<60 \mathrm{kpc}}=4.0 \pm 0.7 \times 10^{11} M_{\odot}$,

which corresponds to $M_{\text {tot }}=1.0_{-0.2}^{+0.3} \times 10^{12} M_{\odot}$. To get this total mass adiabatic contraction (Blumenthal et al. 1986) was allowed in which case the concentration parameter was taken as a free parameter in the fit. This parameter came out to be low $\left(c=6.6_{-1.6}^{+1.8}\right.$; if adiabatic contraction is ignored, the concentration parameter becomes around 12 and the mass decreases to $M_{\text {tot }}=0.9 \times 10^{12} M_{\odot}$, which is well within the errors. Similar mass values were found from the velocity dispersion by Battaglia et al. (2006), although with larger errors. These measurements are consistent with the favoured $\Lambda \mathrm{CDM}$ model 


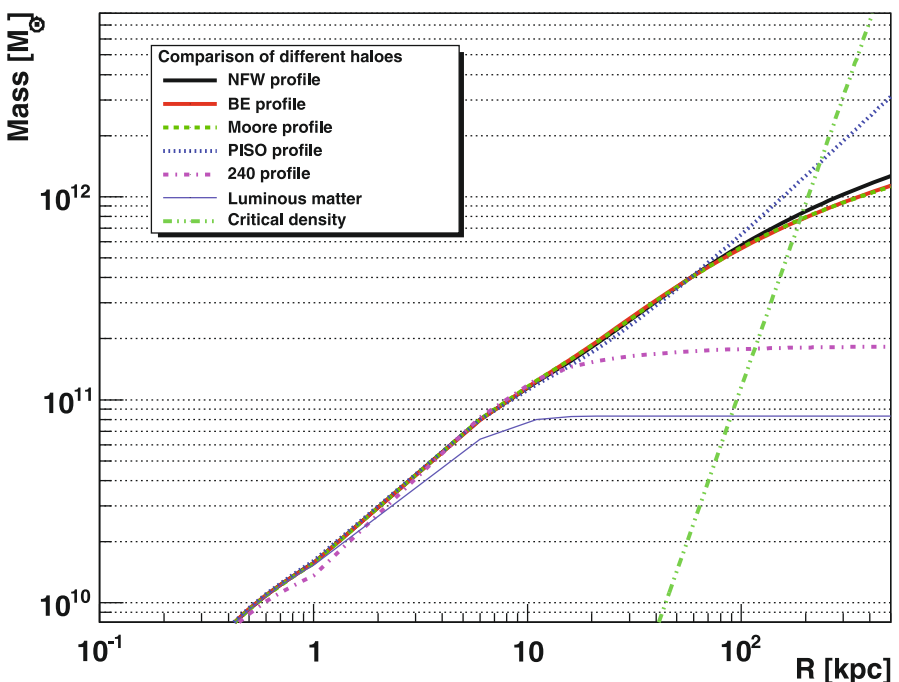

Fig. 2. The mass inside a radius as function of that radius is shown for the different halo profiles defined in Table 1. The thin solid line represents the visible mass which is different for different halo profiles because of the variation of the parameters $\rho_{\mathrm{d}}$ and $r_{\mathrm{d}}$. Here the luminous matter for the NFW profile is shown. The steep line starting at $40 \mathrm{kpc}$ represents the mass of a sphere with a density of 200 times the critical density of the Universe. The crossings of the mass distributions with this line represent the virial radius and the total Galactic mass of the corresponding density distribution.

in Klypin et al. (2002) where the virial mass is $1 \times 10^{12} M_{\odot}$. The value from Eq. (5) will be used in the analysis.

Figure 2 shows the radial dependence of the total Galactic mass for the different spherical halo profiles. At small radii $r<5 \mathrm{kpc}$ the density distribution is dominated by the luminous matter, shown by the thin solid line, which is independent of the halo profile. The mass of a homogeneous sphere with 200 times the critical density of the Universe is also shown. The crossing of a mass distribution with this line defines the total Galactic mass and the virial radius for this density distribution which is $210 \mathrm{kpc}$ for a Galactic mass of $10^{12} M_{\odot}$.

The mass distributions of the NFW and the BE profile are quite similar since these profiles differ only in the region around the GC where the influence of DM is small. The 240 profile yields the smallest mass while the mass distribution of the PISO profile shows a linear increase with radius. The reason is the quadratic decrease $\left(\propto r^{-2}\right)$ of the PISO profile. Consequently, the integral of such a profile leads to a linear increase of the Galactic mass. By going from spherical to elliptical profiles the mass can be changed significantly, as will be discussed later.

\subsection{Rotation curve}

Each object, which is bound to the MW, is orbiting around the GC. Most of the stars and the interstellar medium (gas, dust, etc.) are rotating with a velocity distribution $v(r)$. This velocity distribution is called the rotation curve (RC) of the MW.

For a circular rotation of the objects within the Galactic disc the rotation velocity is given by the equality of the centripetal and the gravitational force

$\frac{m v^{2}}{r}=m \cdot \frac{\mathrm{d} \Phi}{\mathrm{d} r}$ where $v$ is the rotation velocity at the Galactocentric distance $r$. The gravitational potential $\Phi$ is given by the Poisson equation

$\Delta \Phi=-4 \pi G \rho(r)$,

where $G$ is the gravitational constant and $\rho(r)$ is the matter density distribution model. For a given DM density profile the gravitational potential and therefore the rotation curve can be calculated and a comparison with experimental data is an important check for the DM density profile.

The rotation curve can be most easily measured from the Doppler shifts, like the $21 \mathrm{~cm}$ from neutral hydrogen or the rotational transition lines of carbon monoxide (CO) in the millimeter wave range. A review of the various methods was given by Sofue \& Rubin (2001). A recent summary of all data on the rotation curve of the Milky Way can be found in the publication by Sofue et al. (2008), where the numerical values can be found on the author's webpage. For radii inside the solar radius the distances do not need to be determined, since the maximum Doppler shift observed in a given direction is in the tangential direction of the circle, so the longitude of that direction determines the distance from the centre. For radii larger than the solar radius the distances to the tracers have to be determined independently, usually by the angular thickness of the HI layer, as first proposed by Merrifield (1992). These independent distance determinations lead to larger errors in the outer rotation curve.

The most precise determination and with it the normalization of the rotation curve is obtained from the Oort constants, which can be determined from the precise distances and velocities of nearby stars, as discussed in most textbooks, e.g. (Binney 1998; Sparke 2007; Zeilik 1998). These constants are defined as:

$A \equiv-\frac{1}{2}\left[\frac{\mathrm{d} v}{\mathrm{~d} r} \mid r_{\odot}-\frac{v_{\odot}}{r_{\odot}}\right] \approx 14.4 \pm 1.2 \mathrm{~km} \mathrm{~s}^{-1} \mathrm{kpc}^{-1}$
$B \equiv-\frac{1}{2}\left[\frac{\mathrm{d} v}{\mathrm{~d} r} \mid r_{\odot}+\frac{v_{\odot}}{r_{\odot}}\right] \approx-12.0 \pm 2.8 \mathrm{~km} \mathrm{~s}^{-1} \mathrm{kpc}^{-1}$

where the experimental values have been taken from Kerr \& Lynden-Bell (1986). One observes that $A-B=v_{\odot} / r_{\odot}$ defines the local normalization of the rotation curve, while $A+B$ defines the slope at the solar position.

The combination $A-B$ can be more precisely determined than the individual constants. Kerr \& Lynden-Bell (1986) found $A-B=v_{\odot} / r_{\odot}=27 \pm 2.5 \mathrm{~km} \mathrm{~s}^{-1} \mathrm{kpc}^{-1}$.

Using the proper motion of the black hole in the Galactic centre (Sgr A*) Reid \& Brunthaler (2004) found

$A-B=v_{\odot} / r_{\odot}=29.45 \pm 0.15 \mathrm{~km} \mathrm{~s}^{-1} \mathrm{kpc}^{-1}$

in excellent agreement with recent measurements of parallaxes using the Very Large Baseline Interferometry (VLBI) (Reid et al. 2009), which yield $A-B=v_{\odot} / r_{\odot}=30.3 \pm 0.9 \mathrm{~km} \mathrm{~s}^{-1} \mathrm{kpc}^{-1}$. A further interesting property of the RC is its slope at the position of the Sun. This value is strongly connected to the values of $A$ and $B$ and was determined in the publication by Fuchs et al. (2009) to be

$R C_{\text {Slope }}=\frac{\partial \ln \left(v_{\odot}\right)}{\partial \ln (r)}=-\frac{A+B}{A-B}=-0.006 \pm 0.016$.

From the velocities of stars orbiting Sgr A*, which is considered to be the centre of the Galaxy because of its small own velocity, the distance between the Sun and the GC has been determined to (Gillessen et al. 2009):

$r_{\odot}=8.33 \pm 0.35 \mathrm{kpc}$, 


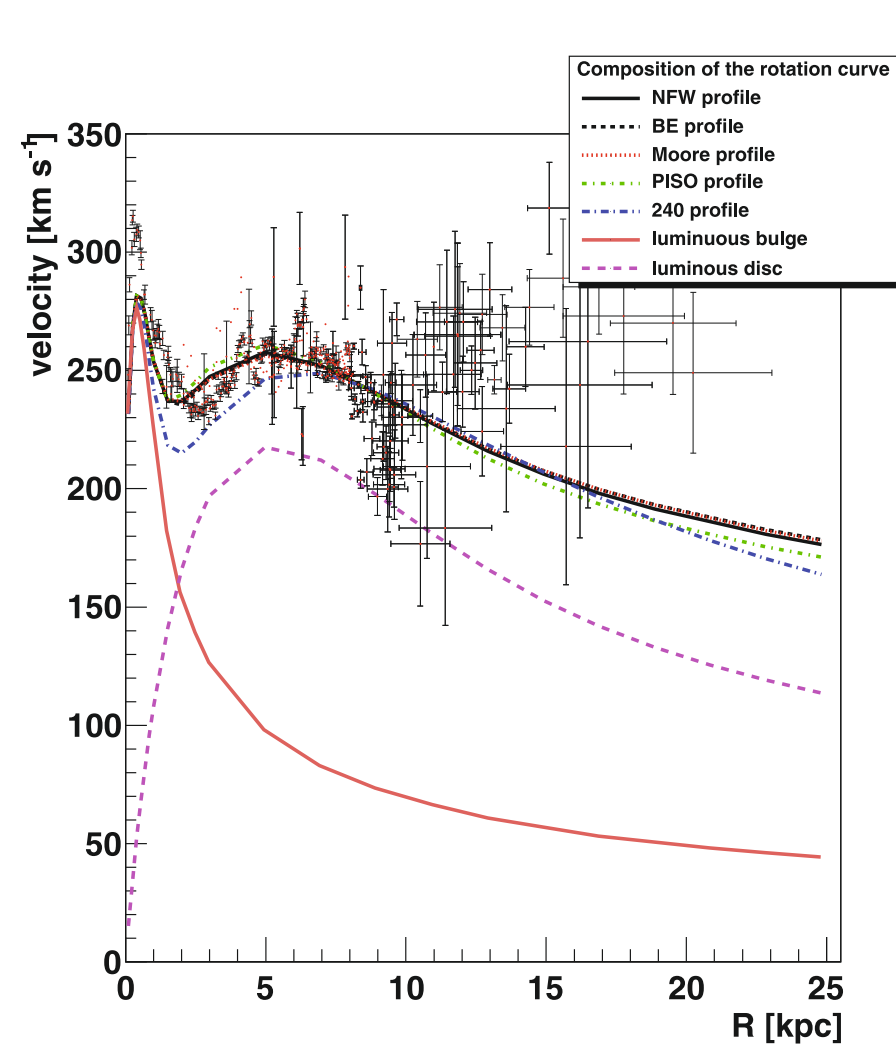

(a) Rotation curve $(z=0 \mathrm{kpc})$, non-averaged

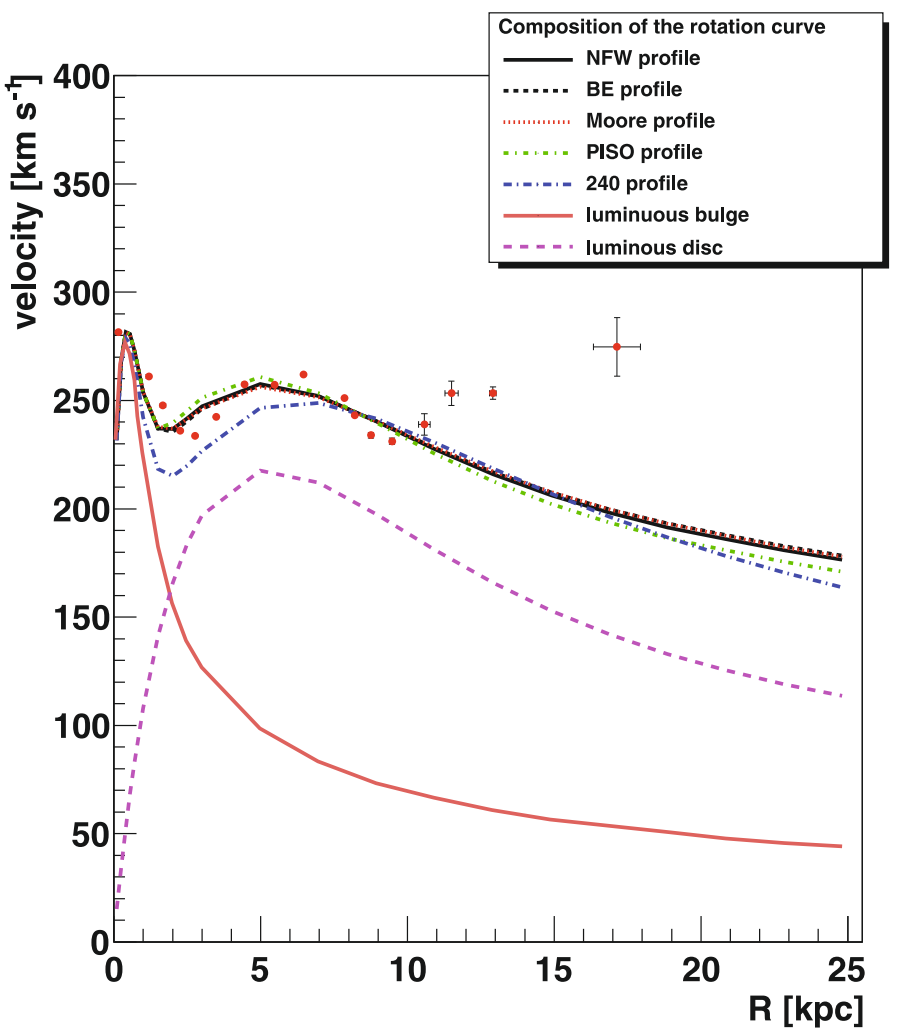

(b) Rotation curve $(z=0 \mathrm{kpc})$, averaged

Fig. 3. The rotation curves - calculated for different halo profiles - in comparison with experimental data, which have been adapted from the publication by Sofue et al. (2008). On the left side all data points are shown, while on the right side a weighted average of the experimental data is shown in 17 radial bins.

in agreement with previous authors (Ghez et al. 2008). With this Galactocentric distance one finds from Eq. (10) a rotation velocity of the Sun

$244 \pm 10 \mathrm{~km} \mathrm{~s}^{-1}$

which is consistent with recent observations of Galactic masers in Bovy et al. (2009), who used data from the Very Long Baseline Array (VLBA) and the Japanese VLBI Exploration of Radio Astronomy (VERA). This speed determines the mass of the Galaxy inside the solar radius.

In this analysis two different rotation velocities are considered: the RC within the Galactic disc (Fig. 3) and the velocity distribution for stars outside the disc with $z>4 \mathrm{kpc}$ (Fig. 4). They are discussed separately.

\subsubsection{Rotation curve in the disc}

For the RC within the disc a combination of different measurements with different tracers has been summarized by Sofue et al. (2008). The experimental data, which can be found on the author's web page, were scaled to $v_{\odot}=244 \mathrm{~km} \mathrm{~s}^{-1}$ at a Galactocentric distance of $8.3 \mathrm{kpc}$. Furthermore, the rotation velocity was averaged in 17 radial bins from the GC to a radius of $22 \mathrm{kpc}$, as shown in Fig. 3b and tabulated in Table 2. The shape of the measured velocity distribution shows a strong increase of the rotation velocity in the inner part of the Galaxy which presumably results from the dense core of the Galaxy. For the inner Galaxy the rotation curve is dominated by the visible matter and the parametrization of Sect. 2.1 yields a reasonable description. However, at the outer Galaxy the experimental data cannot be explained: all profiles predict a slow decrease of the rotation velocity in contrast to the data, which show first a decrease between 6 and $10 \mathrm{kpc}$ and then increases again. Such a peculiar change of slope cannot be explained by a smoothly decreasing DM density profile, but needs substructure, e.g. the infall of a dwarf Galaxy, as mentioned in the introduction and de Boer et al. (2005). Such a ringlike substructure is supported by the gas flaring (Kalberla et al. 2007). The thickness of the substructure is of the order of $1 \mathrm{kpc}$, so it should not show up for halo stars well above this height; this is indeed the case, as shown in Fig. 4, which will be discussed in the next section.

One may argue about the large uncertainties in the outer rotation curve, where the distance and the velocity have to be determined in contrast to the inner rotation curve, where the tangent method yields the distance from the maximum velocity (Binney 1998). The rotation curve can be flattened, e.g. by decreasing the distance between the Sun and the GC, but then $r_{\odot} \approx 7 \mathrm{kpc}$ is needed (Honma \& Sofue 1997), which is clearly outside the present errors given in Eq. (12). Also the peculiar change of slope near $10 \mathrm{kpc}$ does not disappear. It should be noted that such a change of slope happens in other spiral galaxies as well, as can be seen from the compilation of rotation curves in Fig. 4 in Sofue \& Rubin (2001). For the smooth DM density profiles discussed in this paper this feature will be neglected.

\subsubsection{Rotation curve in the halo}

The data in Fig. 4 were obtained from a large sample of roughly 2400 BHB stars, as detected in the SDSS, with Galactocentric distances up to about $60 \mathrm{kpc}$ and vertical heights of $z>4 \mathrm{kpc}$. 


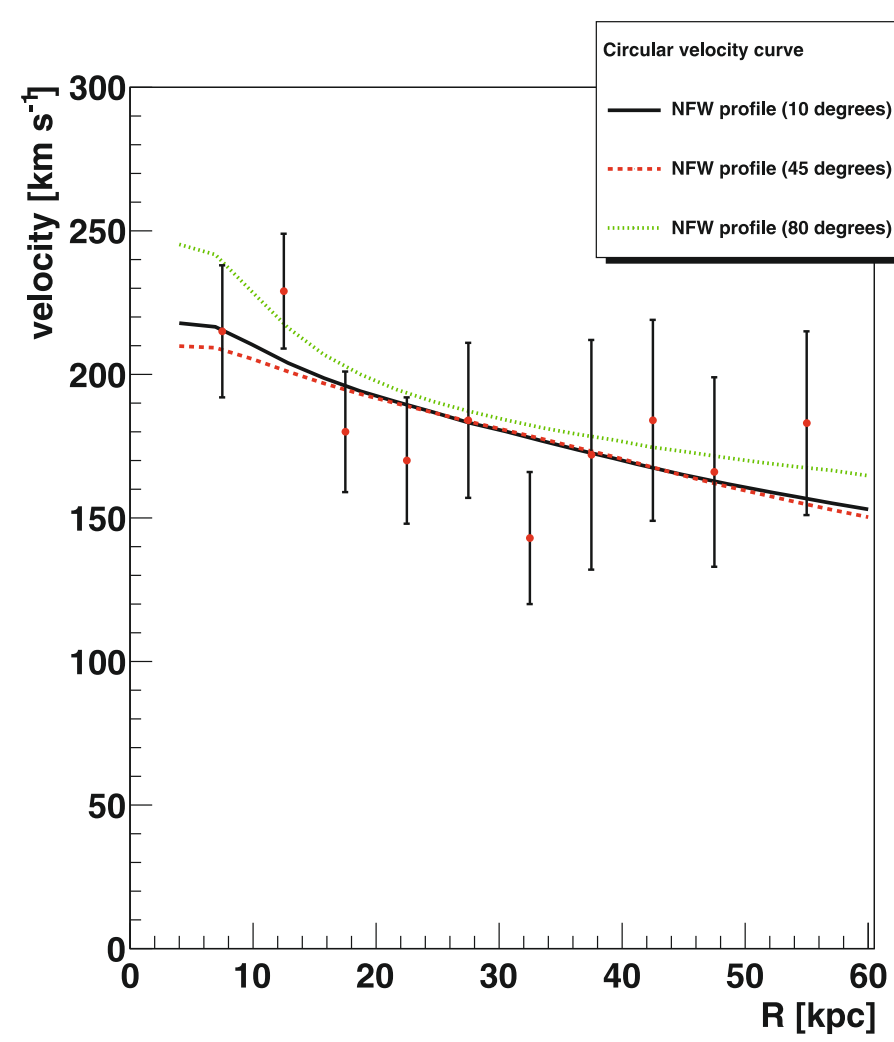

(a) Velocity curve $(z>4 \mathrm{kpc})$, NFW profile

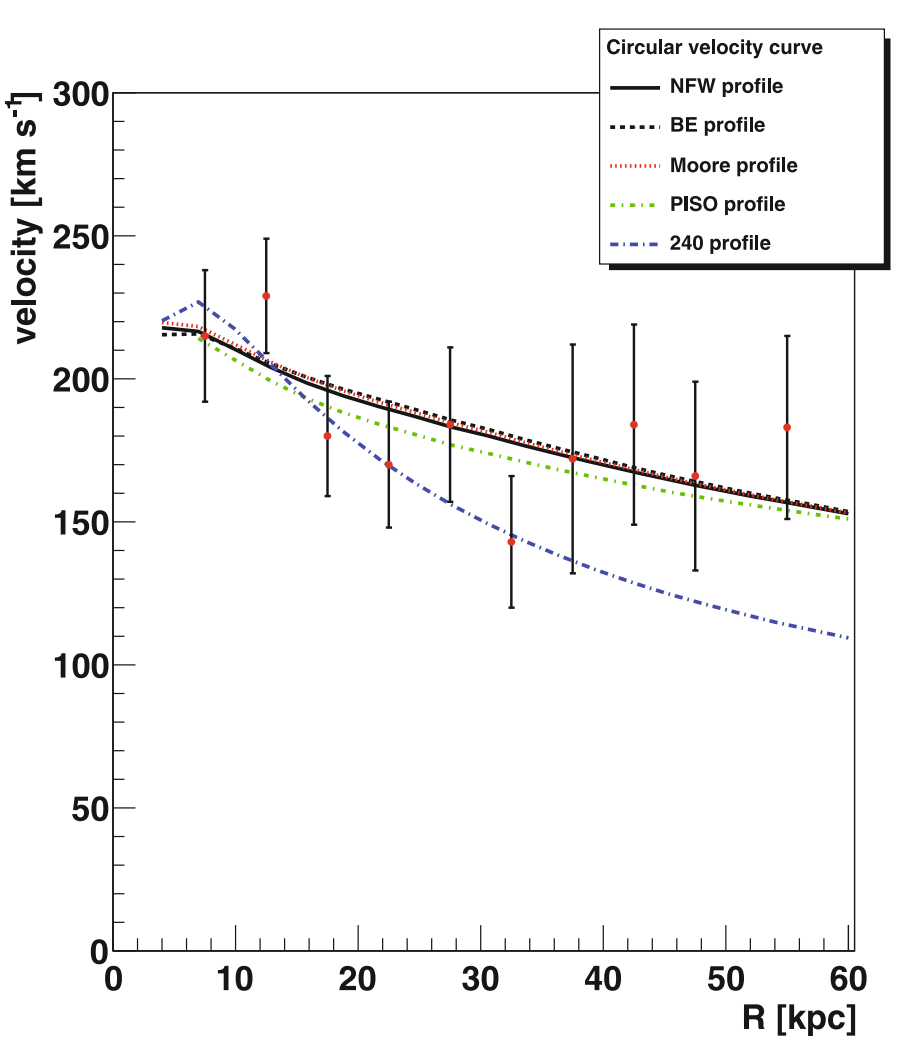

(b) Velocity curve $(z>4 \mathrm{kpc})$ for different DM profiles

Fig. 4. The circular velocity curve for halo stars above a height of $z>4 \mathrm{kpc}$ is shown. On the left side the circular velocity curve for the NFW profile calculated for different angles with respect to the Galactic disc is shown. On the right side the averaged circular velocity curves for the different halo profiles are shown. The experimental data were obtained from the publication by Xue et al. (2008).

Table 2. Averaged values of the Galactocentric distance and the rotation velocity shown in Fig. 3.

\begin{tabular}{l|cccccc}
\hline \hline $\mathrm{Nr}$ & $r_{\text {Min }}$ & $r_{\text {Max }}$ & $r[\mathrm{kpc}]$ & $\sigma_{r}[\mathrm{kpc}]$ & $v\left[\mathrm{~km} \mathrm{~s}^{-1}\right]$ & $\sigma_{v}\left[\mathrm{~km} \mathrm{~s}^{-1}\right]$ \\
\hline 1 & 0.0 & 1.0 & 0.146 & 0.002 & 281.514 & 0.851 \\
2 & 1.0 & 1.5 & 1.190 & 0.011 & 261.127 & 0.783 \\
3 & 1.5 & 2.0 & 1.670 & 0.016 & 247.721 & 0.838 \\
4 & 2.0 & 2.5 & 2.253 & 0.016 & 236.071 & 0.640 \\
5 & 2.5 & 3.0 & 2.770 & 0.020 & 233.645 & 0.474 \\
6 & 3.0 & 4.0 & 3.481 & 0.017 & 242.435 & 0.307 \\
7 & 4.0 & 5.0 & 4.457 & 0.021 & 257.475 & 0.307 \\
8 & 5.0 & 6.0 & 5.481 & 0.026 & 257.255 & 0.321 \\
9 & 6.0 & 7.0 & 6.464 & 0.027 & 261.968 & 0.318 \\
10 & 7.0 & 8.0 & 7.852 & 0.001 & 251.095 & 0.198 \\
11 & 8.0 & 8.5 & 8.202 & 0.009 & 243.078 & 0.352 \\
12 & 8.5 & 9.0 & 8.761 & 0.046 & 233.942 & 1.278 \\
13 & 9.0 & 10.0 & 9.478 & 0.052 & 231.191 & 1.434 \\
14 & 10.0 & 11.0 & 10.578 & 0.199 & 238.947 & 4.963 \\
15 & 11.0 & 12.0 & 11.500 & 0.227 & 253.366 & 5.631 \\
16 & 12.0 & 16.0 & 12.912 & 0.115 & 253.384 & 2.837 \\
17 & 16.0 & 22.0 & 17.141 & 0.796 & 274.735 & 13.594 \\
\hline
\end{tabular}

In order to connect the observable values - line-of-sight velocity and distance - to the circular velocity $v(r)=\sqrt{r \partial \Phi / \partial r}$ the halo star distribution function from $N$-body simulations of the Galaxy with an NFW profile was used.

In order to compare our DM density profiles with these data, the velocity curve is calculated for different angles with respect to the Galactic disc. Then the results are averaged. In Fig. 4 the averaged circular velocity curve and the velocity curves for an inclination angle with the normal to the disc of $10^{\circ}, 45^{\circ}$ and $80^{\circ}$ are shown for the NFW profile. The averaged circular velocity curves for the five other spherical halo profiles discussed before are shown in Fig. 4b. The circular velocity distribution is consistent with the cuspy halo profiles and the PISO profile. The 240 profile cannot describe the velocity distribution at large radii because of the too steep decrease of the density at large radii $\left(\propto 1 / r^{4}\right)$.

\subsection{Surface density and Oort limit}

Jan Oort proposed and performed another interesting measurement: from the star count as function of their height above the disc one obtains the local gravitational potential, which is directly proportional to the mass in the plane of the MW. Using the precise measurements from the Hipparcos satellite Holmberg \& Flynn (2004) find for the local mass density, which includes visible and dark matter,

$\rho_{\odot, \text { tot }}(z=0)=0.102 \pm 0.010 M_{\odot} \mathrm{pc}^{-3}$.

This value was determined by the precise star counts and velocity measurements in a volume of $125 \mathrm{pc}$ around the Sun by the Hipparcos satellite. Korchagin et al. (2003) analysed the vertical potential at slightly larger distances (a vertical cylinder of $200 \mathrm{pc}$ radius and an extension of $400 \mathrm{pc}$ out of the Galactic plane). For the dynamical estimate of the local volume density they obtain the same value with a smaller error: $\rho_{\odot, \text { tot }}(z=0)=0.100 \pm 0.005 M_{\odot} \mathrm{pc}^{3}$. To be conservative, we 
Table 3. Contributions to the local surface density of baryonic matter. The total values in the last row include $\pm 1 \sigma$ errors.

\begin{tabular}{c|c|c}
\hline \hline Contribution & $\Sigma\left[M_{\odot} \mathrm{pc}^{-2}\right]$ & Reference \\
\hline Visible stars & $35 \pm 5$ & Gilmore et al. (1989) \\
& 27 & Gould et al. (1996) \\
& 30 & Zheng et al. (2001) \\
\hline Stellar remnants & $3 \pm 1$ & Mera et al. (1998) \\
\hline Interstellar gas & $8 \pm 5$ & Dame (1993) \\
\hline & $13-14$ & Olling \& Merrifield (2001) \\
\hline Total & $35-58$ & \\
\hline
\end{tabular}

will use the value from Eq. (14). It is called the Oort limit, since it gives the lowest value for the local density.

Integrating the density along the vertical direction within $\pm z$ from the Galactic plane yields the surface density:

$\Sigma_{\text {def }}(<|z|)=\int_{-z}^{z} \rho\left(z^{\prime}\right) \mathrm{d} z^{\prime}$.

It can be calculated either by integrating the matter density distribution directly or by using the gravitational potential of the Galaxy. The integration limit is conventionally defined to be $1.1 \mathrm{kpc}$.

According to Eq. (7) this definition is equal to

$$
\begin{aligned}
\Sigma_{\mathrm{pot}}(<|z|) & =\frac{1}{2 \pi G} \cdot \int_{0}^{z} \Delta \Phi\left(r, \varphi, z^{\prime}\right) \mathrm{d} z^{\prime} \\
& =\frac{1}{2 \pi G} \cdot \int_{0}^{z}\left(\frac{1}{r} \frac{\partial}{\partial r}\left(r \frac{\partial \Phi}{\partial r}\right)+\frac{1}{r^{2}} \frac{\partial^{2} \Phi}{\partial \varphi^{2}}+\frac{\partial^{2} \Phi}{\partial z^{\prime 2}}\right) \mathrm{d} z^{\prime} \\
& =\frac{1}{2 \pi G} \cdot\left(\frac{\partial \Phi}{\partial z}+\int_{0}^{z}\left(\frac{1}{r} \frac{\partial}{\partial r}\left(r \frac{\partial \Phi}{\partial r}\right)+\frac{1}{r^{2}} \frac{\partial^{2} \Phi}{\partial \varphi^{2}}\right) \mathrm{d} z^{\prime}\right) \\
& =\frac{1}{2 \pi G} \cdot\left(\frac{\partial \Phi}{\partial z}+2\left(A^{2}-B^{2}\right)|z|\right) \\
& \approx \frac{1}{2 \pi G} \cdot\left(\frac{\partial \Phi}{\partial z}\right)
\end{aligned}
$$

where $A$ and $B$ are the Oort constants discussed before. Since they are of the same order of magnitude, the surface density integrated to $z$ is proportional to the derivative of the potential at height $z$, as shown by the last approximation in Eq. (16).

First the surface density of the luminous matter is considered. Its experimental value is determined by the summation of the different contributions to the luminous matter - the stellar population, stellar remnants and the interstellar gas. A summary of the different measurements was given by Naab \& Ostriker (2006). The surface density of the baryonic matter lies between 35 and $58 M_{\odot} \mathrm{pc}^{-2}$ (Table 3 ), which agrees with $48 \pm 9 M_{\odot} \mathrm{pc}^{-2}$ as estimated in Kuijken \& Gilmore (1991) and Holmberg \& Flynn (2004).

Unfortunately, our local neighbourhood is not representative for the disc, since we live in a local underdensity - the local bubble - with an extension of a few hundred pc, which could have been caused by a series of rather recent $\mathrm{SN}$ explosions (Maiz-Apellaniz 2001). Therefore, a fit for the parameters of a mass model of the Galaxy might have a somewhat higher surface density than the locally observed value.

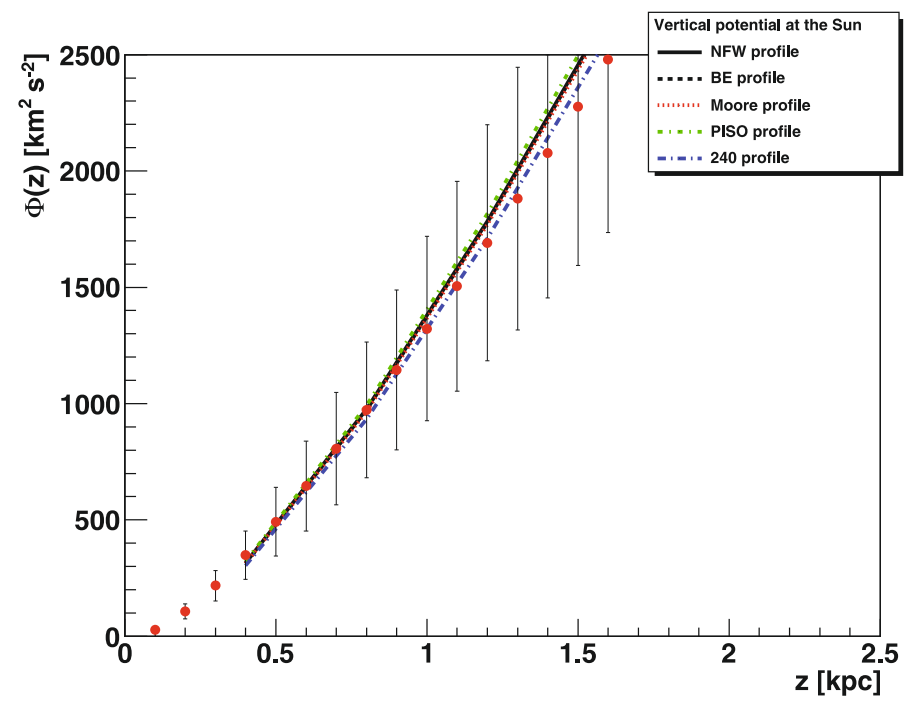

Fig. 5. The vertical gravitational potential at the position of the Sun $\Phi(z)$. The experimental data is adapted from (Bienayme et al. 2005) and the errors are assumed to be $30 \%$.

The total surface density at the position of the Sun was determined by Kuijken \& Gilmore (1991) to be

$\Sigma(<1.1 \mathrm{kpc})=71 \pm 6 M_{\odot} \mathrm{pc}^{-2}$

from a parametrization of a mass distribution. In the paper by Holmberg \& Flynn (2004) the modeling of the vertical gravitational potential resulted in $\Sigma(<1.1 \mathrm{kpc})=74 \pm 6 M_{\odot} \mathrm{pc}^{-2}$. The most recent determination of the surface density by Bienayme et al. (2005) is consistent with both measurements but allows somewhat larger errors: $\Sigma(<1.1 \mathrm{kpc})=68 \pm 11 M_{\odot} \mathrm{pc}^{-2}$. Comparing this total surface density with the baryonic surface density shown in Table 3 , it is clear that the local gravitational potential is largely determined by the stars, so it will be practically independent of the DM halo profile, as shown in Fig. 5 for different halo profiles.

\section{Numerical determination of the mass model of the Galaxy}

As mentioned before, the three most important constraints for the mass model of the Galaxy are given by the rotation curve and the value $v_{\odot}$, the total mass $M_{\mathrm{tot}}$ and the local mass density $\rho_{\odot, \text { tot }}$. This can be easily seen as follows: $v_{\odot}^{2}=v_{\mathrm{vis}}^{2}+v_{\mathrm{DM}}^{2}$, where $v_{\mathrm{vis}}^{2}$ and $v_{\mathrm{DM}}^{2}$ are proportional to $\rho_{\mathrm{vis}}$ and $\rho_{\mathrm{DM}}$, respectively; for a given halo profile $M_{\text {tot }}$ is determined by $\rho_{\text {DM }}$, while the Oort limit $\rho_{\odot, \text { tot }}$ determines $\rho_{\odot, \text { tot }}=\rho_{\text {vis }}+\rho_{\text {DM }}$. So in principle one has 3 constraints with only 2 variables $\rho_{\mathrm{vis}}$ and $\rho_{\mathrm{DM}}$, if the shapes of the DM halo and the visible matter would be known.

Unfortunately, additional important parameters are i) the eccentricity of the DM halo ii) the concentration of the DM halo iii) the scale length of the disc iv) the mass in the bar/bulge. In addition the mass model is sensitive to the geometry, i.e. the Galactocentric distance from the Sun $d_{\odot}$ and the halo profile. Additional constraints come from the surface density, but here the visible surface density has a large uncertainty as discussed before. The parameters and constraints have been summarized in Table 4. The parametrization of the mass of the bulge was chosen to describe the rotation curve at small radii, which works reasonably well, as can be seen from Fig. 3. Given that the mass model is not very sensitive to this inner region, the parameters of the bulge will not be varied anymore. 
Table 4. Free and fixed parameters for the mass model of the Galaxy and experimental constraints. One observes that there are 5 free parameters and 8 constraints. Mass densities are in $\mathrm{GeV} \mathrm{cm}{ }^{-3}$ or in $M_{\odot} \mathrm{pc}^{-3}$, where $1 M_{\odot} \mathrm{cm}^{-3} \equiv 37.97 \mathrm{GeV} \mathrm{cm}^{-3}$.

\begin{tabular}{|c|c|c|c|c|}
\hline \multicolumn{5}{|c|}{ Free parameters } \\
\hline Component & Parameter & Symbol & Value & Unit \\
\hline Halo & Local DM density & $\rho_{\odot, \mathrm{DM}}$ & - & $\mathrm{GeV} / \mathrm{cm}^{-3}$ \\
\hline Halo & Scale Parameter & $a$ & - & $\mathrm{kpc}$ \\
\hline Halo & eccentricity & $\epsilon_{z}$ & - & $\mathrm{kpc}$ \\
\hline Disc & Density at GC & $\rho_{\mathrm{d}}$ & - & $\mathrm{GeV} \mathrm{cm}^{-3}$ \\
\hline Disc & scale length & $r_{\mathrm{d}}$ & - & $\mathrm{kpc}$ \\
\hline \multicolumn{5}{|c|}{ Fixed parameters } \\
\hline Disc & scale height & $z_{\mathrm{d}}$ & 320 & $\mathrm{pc}$ \\
\hline Bulge & Density at GC & $\rho_{\mathrm{b}}$ & 360 & $\mathrm{GeV} \mathrm{cm}^{-3}$ \\
\hline Bulge & Eccentricity & $q_{\mathrm{b}}$ & 0.61 & \\
\hline Bulge & Scale & $r_{\mathrm{t}}$ & 0.6 & $\mathrm{kpc}$ \\
\hline Bulge & Scale & $r_{0, \mathrm{~b}}$ & 0.7 & $\mathrm{kpc}$ \\
\hline Bulge & Slope & $\gamma_{\mathrm{b}}$ & 1.6 & \\
\hline Bulge & Slope & $\beta_{\mathrm{b}}$ & 1.6 & \\
\hline \multicolumn{5}{|c|}{ Constraints } \\
\hline All & Mass inside $60 \mathrm{kpc}$ & $M_{R<60 \mathrm{kpc}}$ & $4.0 \pm 0.7$ & $10^{11} M_{\odot}$ \\
\hline Local & Rotation speed Sun & $v_{\odot}$ & $244 \pm 10$ & $\mathrm{~km} \mathrm{~s}^{-1}$ \\
\hline Local & Distance Sun-GC & $r_{\odot}$ & $8.33 \pm 0.35$ & $\mathrm{kpc}$ \\
\hline Local & Total Surface Density & $\Sigma_{|z|<1.1}$ & $71 \pm 6$ & $M_{\odot} \mathrm{pc}^{-2}$ \\
\hline Local & Visible Surface Density & $\sum_{\text {vis }}$ & $48 \pm 9$ & $M_{\odot} \mathrm{pc}^{-2}$ \\
\hline Local & Mass Density & $\rho_{\text {tot }}$ & $0.102 \pm 0.01$ & $M_{\odot} \mathrm{pc}^{-3}$ \\
\hline Local & Oort Constants & $A-B$ & $29.45 \pm 0.15$ & $\mathrm{~km} \mathrm{~s}^{-2} \mathrm{kpc}^{-1}$ \\
\hline Local & Slope of rotation curve & $\partial \ln \left(v_{\odot}\right) / \partial \ln (r)$ & $-0.006 \pm 0.016$ & \\
\hline
\end{tabular}

Table 5. Fit results. The units of the different values are given in Table 4 . The $\chi^{2}$ contributions are given below the variable value in brackets.

\begin{tabular}{|c|c|c|c|c|c|c|c|c|c|c|c|c|c|c|c|}
\hline \multirow[b]{2}{*}{$\mathrm{Nr}$} & \multirow[b]{2}{*}{ Profile } & \multicolumn{3}{|c|}{ Fitted parameters } & \multicolumn{11}{|c|}{ Derived quantities } \\
\hline & & $\rho_{\odot, \mathrm{DM}}$ & $a$ & $\rho_{\mathrm{d}}$ & $r_{\mathrm{d}}$ & $c_{\mathrm{vir}}$ & $\rho_{\odot, \text { tot }}$ & $v_{\odot}$ & $M_{60}$ & $M_{\mathrm{tot}}$ & $A-B$ & $\frac{\partial \ln \left(v_{\odot}\right)}{\partial \ln (r)}$ & $\Sigma_{|z|<1.1}$ & $\Sigma_{\text {vis }}$ & $x^{2}$ \\
\hline 1 & NFW & $0.32 \pm 0.05$ & 10 & $88.3 \pm 19.8$ & $\begin{array}{c}2.5 \pm 0.2 \\
(0.13)\end{array}$ & 17.5 & $\begin{array}{l}0.094 \\
(0.58)\end{array}$ & $\begin{array}{c}244.5 \\
(0.0)\end{array}$ & $\begin{array}{c}3.9 \times 10^{11} \\
(0.0)\end{array}$ & $6.5 \times 10^{11}$ & $\begin{array}{l}29.45 \\
(0.01)\end{array}$ & $\begin{array}{c}-0.002 \\
(0.06)\end{array}$ & $\begin{array}{c}72.2 \\
(0.04)\end{array}$ & $\begin{array}{c}53.8 \\
(0.41)\end{array}$ & $(1.25)$ \\
\hline 2 & NFW & $0.27 \pm 0.06$ & 15 & $113.1 \pm 17.1$ & $\begin{array}{c}2.4 \pm 0.1 \\
(0.01)\end{array}$ & 12.0 & $\begin{array}{l}0.096 \\
(0.38)\end{array}$ & $\begin{array}{l}244.5 \\
(0.0)\end{array}$ & $\begin{array}{c}4.0 \times 10^{11} \\
(0.0)\end{array}$ & $7.4 \times 10^{11}$ & $\begin{array}{c}29.45 \\
(0.0)\end{array}$ & $\begin{array}{c}-0.003 \\
(0.04)\end{array}$ & $\begin{array}{l}71.1 \\
(0.0)\end{array}$ & $\begin{array}{c}55.4 \\
(0.68)\end{array}$ & (1.13) \\
\hline 3 & NFW & $0.23 \pm 0.05$ & 20 & $128.7 \pm 69.8$ & $\begin{array}{c}2.3 \pm 0.1 \\
(0.0)\end{array}$ & 9.5 & $\begin{array}{l}0.097 \\
(0.27) \\
\end{array}$ & $\begin{array}{c}244.4 \\
(0.0) \\
\end{array}$ & $\begin{array}{c}4.1 \times 10^{11} \\
(0.02)\end{array}$ & $8.3 \times 10^{11}$ & $\begin{array}{r}29.45 \\
(0.0) \\
\end{array}$ & $\begin{array}{r}-0.003 \\
(0.03)\end{array}$ & $\begin{array}{c}70.2 \\
(0.02) \\
\end{array}$ & $\begin{array}{r}56.6 \\
(0.92) \\
\end{array}$ & $(1.27)$ \\
\hline 4 & NFW & $0.32 \pm 0.04$ & $10.8 \pm 3.4$ & $91.0 \pm 8.0$ & 2.5 & 16.2 & $\begin{array}{l}0.095 \\
(0.50)\end{array}$ & $\begin{array}{c}244.4 \\
(0.0)\end{array}$ & $\begin{array}{c}4.0 \times 10^{11} \\
(0.0)\end{array}$ & $6.7 \times 10^{11}$ & $\begin{array}{c}29.45 \\
(0.0)\end{array}$ & $\begin{array}{c}-0.003 \\
(0.04)\end{array}$ & $\begin{array}{c}72.4 \\
(0.06)\end{array}$ & $\begin{array}{c}54.2 \\
(0.48)\end{array}$ & (1.07) \\
\hline 5 & NFW & $0.35 \pm 0.06$ & $14.9 \pm 4.8$ & $89.5 \pm 8.2$ & 2.5 & 13.4 & $\begin{array}{l}0.094 \\
(0.57)\end{array}$ & $\begin{array}{c}244.4 \\
(0.0)\end{array}$ & $4.25 \times 10^{11}$ & $\begin{array}{c}9.8 \times 10^{11} \\
(0.0)\end{array}$ & $\begin{array}{c}29.45 \\
(0.0)\end{array}$ & $\begin{array}{c}-0.003 \\
(0.05)\end{array}$ & $\begin{array}{c}73.7 \\
(0.21)\end{array}$ & $\begin{array}{c}53.2 \\
(0.34)\end{array}$ & (1.17) \\
\hline 6 & NFW & $0.39 \pm 0.05$ & $20.4 \pm 4.5$ & $88.1 \pm 8.7$ & 2.5 & 11.5 & $\begin{array}{l}0.094 \\
(0.62)\end{array}$ & $\begin{array}{l}244.4 \\
(0.0)\end{array}$ & $5.5 \times 10^{11}$ & $\begin{array}{c}1.49 \times 10^{12} \\
(0.0)\end{array}$ & $\begin{array}{l}29.44 \\
(0.08)\end{array}$ & $\begin{array}{c}-0.001 \\
(0.0)\end{array}$ & $\begin{array}{c}75.0 \\
(0.45)\end{array}$ & $\begin{array}{c}52.4 \\
(0.24)\end{array}$ & $(1.40)$ \\
\hline 7 & NFW & $0.41 \pm 0.05$ & $25.2 \pm 4.6$ & $87.3 \pm 8.9$ & 2.5 & 10.1 & $\begin{array}{l}0.094 \\
(0.64)\end{array}$ & $\begin{array}{l}244.4 \\
(0.0)\end{array}$ & $6.5 \times 10^{11}$ & $\begin{array}{c}1.98 \times 10^{12} \\
(0.0)\end{array}$ & $\begin{array}{c}29.44 \\
(0.0)\end{array}$ & $\begin{array}{c}-0.001 \\
(0.09)\end{array}$ & $\begin{array}{c}75.9 \\
(0.68)\end{array}$ & $\begin{array}{c}51.9 \\
(0.19)\end{array}$ & (1.61) \\
\hline 8 & $\mathrm{BE}$ & $0.25 \pm 0.05$ & 10.2 & $133.6 \pm 15.2$ & $\begin{array}{c}2.29 \pm 0.09 \\
(0.0)\end{array}$ & 17.6 & $\begin{array}{l}0.096 \\
(0.31)\end{array}$ & $\begin{array}{c}244.4 \\
(0.0)\end{array}$ & $\begin{array}{c}4.1 \times 10^{11} \\
(0.01)\end{array}$ & $7.5 \times 10^{11}$ & $\begin{array}{l}29.45 \\
(0.0)\end{array}$ & $\begin{array}{c}-0.003 \\
(0.03)\end{array}$ & $\begin{array}{c}70.6 \\
(0.01)\end{array}$ & $\begin{array}{c}56.2 \\
(0.84)\end{array}$ & $(1.20)$ \\
\hline 9 & Moore & $0.25 \pm 0.05$ & 30.0 & $114.7 \pm 17.3$ & $\begin{array}{c}2.36 \pm 0.11 \\
(0.01)\end{array}$ & 6.2 & $\begin{array}{l}0.095 \\
(0.32)\end{array}$ & $\begin{array}{l}244.4 \\
(0.0)\end{array}$ & $\begin{array}{c}4.1 \times 10^{11} \\
(0.01)\end{array}$ & $7.6 \times 10^{11}$ & $\begin{array}{c}29.45 \\
(0.0)\end{array}$ & $\begin{array}{c}-0.003 \\
(0.04)\end{array}$ & $\begin{array}{c}70.4 \\
(0.01)\end{array}$ & $\begin{array}{c}56.2 \\
(0.82)\end{array}$ & $(1.21)$ \\
\hline 10 & PISO & $0.20 \pm 0.04$ & 5.0 & $150.4 \pm 12.9$ & $\begin{array}{c}2.21 \pm 0.08 \\
(0.02)\end{array}$ & 46 & $\begin{array}{l}0.098 \\
(0.19)\end{array}$ & $\begin{array}{c}244.4 \\
(0.0)\end{array}$ & $\begin{array}{c}4.1 \times 10^{11} \\
(0.03)\end{array}$ & $1.45 \times 10^{12}$ & $\begin{array}{c}29.45 \\
(0.0)\end{array}$ & $\begin{array}{c}-0.004 \\
(0.02)\end{array}$ & $\begin{array}{c}69.3 \\
(0.08)\end{array}$ & $\begin{array}{c}57.8 \\
(1.18)\end{array}$ & (1.54) \\
\hline 11 & 240 & $0.26 \pm 0.03$ & 4.0 & $53.1 \pm 9.6$ & $\begin{array}{c}3.0 \pm 0.2 \\
(1.36)\end{array}$ & 26.3 & $\begin{array}{l}0.095 \\
(0.51)\end{array}$ & $\begin{array}{l}244.6 \\
(0.0)\end{array}$ & $\begin{array}{c}1.7 \times 10^{11} \\
(10.45)\end{array}$ & $1.8 \times 10^{11}$ & $\begin{array}{l}29.47 \\
(0.02)\end{array}$ & $\begin{array}{c}-0.002 \\
(0.06)\end{array}$ & $\begin{array}{c}70.0 \\
(0.03)\end{array}$ & $\begin{array}{c}55.0 \\
(0.61)\end{array}$ & (13.04) \\
\hline 12 & NFW & $0.52 \pm 0.07$ & $26.6 \pm 4.9$ & $84.2 \pm 9.5$ & 2.5 & 9.6 & $\begin{array}{l}0.094 \\
(0.66)\end{array}$ & $\begin{array}{l}244.3 \\
(0.01)\end{array}$ & $6.6 \times 10^{11}$ & $\begin{array}{c}1.95 \times 10^{12} \\
(0.03)\end{array}$ & $\begin{array}{l}29.43 \\
(0.02)\end{array}$ & $\begin{array}{c}-0.001 \\
(0.10)\end{array}$ & $\begin{array}{c}80.3 \\
(2.38)\end{array}$ & $\begin{array}{c}50.1 \\
(0.66)\end{array}$ & (3.24) \\
\hline
\end{tabular}

To optimize the remaining parameters in order to best describe the data, the following $\chi^{2}$ function was minimized using the Minuit package (James \& Roos 1975)

$$
\begin{aligned}
\chi^{2}= & \frac{\left(M_{\mathrm{tot}}^{\mathrm{calc}}-D\right)^{2}}{\sigma_{M_{\mathrm{tot}}}^{2}}+\frac{\left(v_{\odot}^{\mathrm{calc}}-D\right)^{2}}{\sigma_{v_{\odot}}^{2}}+\frac{\left(\rho_{\mathrm{tot}}^{\mathrm{calc}}-D\right)^{2}}{\sigma_{\rho_{\mathrm{tot}}}^{2}} \\
& +\frac{\left(\Sigma_{\mathrm{vis}}^{\mathrm{calc}}-D\right)^{2}}{\sigma_{\Sigma_{\mathrm{vis}}}^{2}}+\frac{\left(\Sigma_{\mathrm{tot}}^{\mathrm{calc}}-D\right)^{2}}{\sigma_{\Sigma_{\mathrm{tot}}}^{2}}+\frac{\left(r_{\mathrm{d}}^{\mathrm{calc}}-D\right)^{2}}{\sigma_{r_{\mathrm{d}}}^{2}} \\
& +\frac{\left(R C_{\text {Slope }}^{\text {calc }}-D\right)^{2}}{\sigma_{R C_{\text {Slope }}}^{2}}+\frac{\left((A-B)^{\mathrm{calc}}-D\right)^{2}}{\sigma_{A-B}^{2}} .
\end{aligned}
$$

The index ${ }^{\text {calc }}$ means the observables were calculated from the fitted parameters, while $D$ denotes the experimental data for the observable and $\sigma$ its error. The constraints have been summarized in Table 4.

The fit shows a more than $95 \%$ positive correlation between the local dark matter density and the scale length of DM halo a and an equally large negative correlation with the scale length $r_{\mathrm{d}}$ of the baryonic disc. Consequently, it is difficult to leave parameters free in the fit. Therefore the fit was first performed for fixed values of a (rows 1-3 of Table 5) and then $r_{\mathrm{d}}$ was fixed (rows 47). With the other free parameters all experimental constraints could be met, as indicated by the $\chi^{2}$ values in brackets below the fitted values in Table 5. Of course, the total mass changed for the different fits. Figure 6 shows the resulting local DM density 


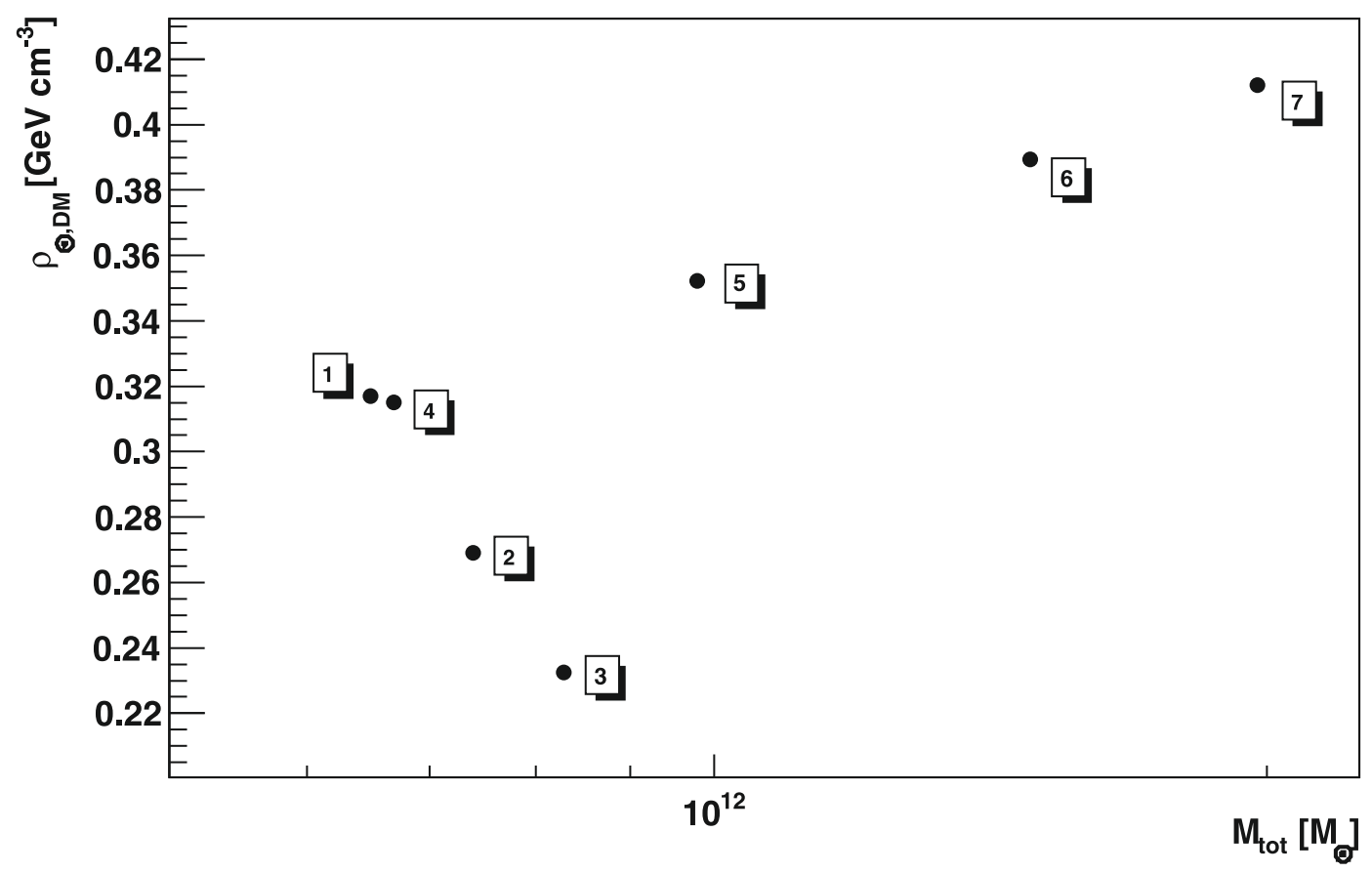

Fig. 6. The local DM densities $\rho_{\odot, \mathrm{DM}}$ are shown for different fits with different parameters. The numbers correspond to the numbers of the fit results in Table 5.

versus the total mass, as calculated from the fitted parameters. It shows that in spite of the small errors for the local density in individual fits the spread in density is still quite large.

The fit was repeated for other halo profiles, which gave similarly good $\chi^{2}$ values, as shown by rows $9-11$ in Table 5 . So with the present data one cannot distinguish the different halo profiles.

Sofar only spherical haloes have been discussed. Allowing oblate haloes with a ratio of short-to-long axis of 0.7 the local DM density increases by about $20 \%$, as shown by the last row of Table 5. As mentioned before, dark discs can enhance this value considerably more, so the uncertainty usually quoted for the local dark matter density in the range of 0.2 to $0.7 \mathrm{GeV} \mathrm{cm}^{-3}$ (0.005-0.018 $\left.M_{\odot} \mathrm{pc}^{-3}\right)$ (Amsler et al. 2008; Gates et al. 1995) is still valid in spite of the considerably improved data.

\section{Conclusion}

In this analysis five different halo profiles are compared with recent dynamical constraints as summarized in Table 4. The change of slope in the RC around $10 \mathrm{kpc}$ (Fig. 3) was ignored, so the monotonical decreasing RC for the smooth halo profiles do not describe the data well. The change of slope may be related to a ringlike DM substructure, as indicated by the structure in the gas flaring (Kalberla et al. 2007) and by the structure in the diffuse gamma radiation (de Boer et al. 2005). Such a ringlike structure of DM gives a perfect description of the rotation curve, especially the fast decrease between 6 and $10 \mathrm{kpc}$. If the DM substructure is included, the local DM density increases above the values found in this analysis, so the values quoted here should be considered lower limits.

The astronomical constraints are consistent with a density model of the Galaxy consisting of a central bulge, a disc and an extended DM halo with a cuspy density profile and a local DM density between $0.2 \mathrm{GeV} \mathrm{cm}^{-3}\left(0.005 M_{\odot} \mathrm{pc}^{-3}\right)$ and $0.4 \mathrm{GeV} \mathrm{cm} \mathrm{cm}^{-3}\left(0.01 M_{\odot} \mathrm{pc}^{-3}\right)$, as shown in Fig. 6. Strong positive and negative correlations between the parameters were found in the fit and they are causing the obvious correlations between $\rho_{\odot, \mathrm{DM}}$ and $M_{\text {tot }}$ in Fig. 6 . For non-spherical haloes these values can be enhanced by $20 \%$. If dark discs are considered, densities up to $0.7 \mathrm{GeV} \mathrm{cm}{ }^{-3}\left(0.018 M_{\odot} \mathrm{pc}^{-3}\right)$ can be easily imagined, so the previous quoted range of $0.2-0.7 \mathrm{GeV} \mathrm{cm}^{-3}$ $\left(0.005-0.018 M_{\odot} \mathrm{pc}^{-3}\right)$ seems still valid. This range is considerably larger than the values quoted by analyses which used a Markov Chain method to minimize the likelihood; they find $\rho_{\odot, \mathrm{DM}}=0.39 \pm 0.03 \mathrm{GeV} \mathrm{cm}^{-3}$ (Catena \& Ullio 2009) and $\rho_{\odot, \mathrm{DM}}=0.32 \pm 0.07 \mathrm{GeV} \mathrm{cm}^{-3}$ (Strigari \& Trotta 2009) respectively. But given the good $\chi^{2}$ values for our fits obtained for a large range of DM densities we see no way that the errors can be as small as quoted by these authors.

\section{References}

Amsler, C., Doser, M., Antonelli, M., et al. 2008, Phys. Lett., B 667, 1 Battaglia, G., Helmi, A., Morrsion, H., et al. 2005, MNRAS, 364, 433 Battaglia, G., Helmi, A., Morrsion, H., et al. 2006, MNRAS, 370, 1055 Bekenstein, J., \& Milgrom, M. 1984, AJ, 286, 7

Bienayme, O., Soubiran, C., Mishenina, T. V., Kovtyukh, V. V., \& Siebert, A. 2005, A\&A, 446, 933

Bienayme, O., Famaey, B., Wu, X., Zhao, H. S., \& Aubert, D. 2009, A\&A, 500, 801

Binney, J. J., \& Evans, N. W. 2001, MNRAS, 327, L27

Binney, E., \& Merrifield, M. R. 1998, Galactic astronomy (Princeton University Press)

Blumenthal, G. R., Faber, S. M., Flores, R., \& Primack, J. R. 1986, ApJ, 301, 27

Bovy, J., Hogg, D. W., \& Rix, H.-W. 2009, ApJ, 704, 1704

Cardone, V. F., \& Sereno, M. 2005, A\&A, 438, 545

Catena, R., \& Ullio, P. 2009, JCAP, submitted

Dame, T. M. 1993, in Back to the Galaxy, ed. S. S. Holt, \& F. Verter, AIP Conf., 278,267

de Boer, W., Sander, C., Zhukov, V., Gladyshev, A. V., \& Kazakov, D. I. 2005, A\&A, 444, 51

Freudenreich, H. T. 1998, ApJ, 492, 495

Fuchs, B., Dettbarn, C., Rix, H.-W., et al. 2009, AJ, 137, 4149

Gates, E. I., Gyuk, G., \& Turner, M. S. 1995, ApJ, 449, L123

Gentile, G., Tonini, C., \& Salucci, P. 2007, A\&A, 467, 925

Ghez, A. M., Salim, S., Weinberg, N. N., et al. 2008, ApJ, 689, 1044

Gillessen, S., Eisenhauer, F., Trippe, S., et al. 2009, ApJ, 692, 1075

Gilmore, G., \& Reid, N. 1983, MNRAS, 202, 1025 
Gilmore, G., Wyse, R. F. G., \& Kuijken, K. 1989, in Evolutionary phenomena in galaxies (Cambridge University Press), 172

Gould, A., Bahcall, J. N., \& Flynn, C. 1996, ApJ, 465, 759

Gunn, J. E. 1977, ApJ, 218, 592

Hammer, F., Puech, M., Chemin, L., Flores, H., \& Lehnert, M. 2007, ApJ, 662, 322

Holmberg, J., \& Flynn, C. 2004, MNRAS, 352, 440

Honma, M., \& Sofue, Y. 1997, Astron. Soc. Japan, 48, L103

James, F., \& Roos, M. 1975, Comput. Phys. Commun., 10, 343

Kalberla, P. M. W., Dedes, L., Kerp, J., \& Haud, U. 2007, A\&A, 469, 511

Kerr, F. J., \& Lynden-Bell, D. 1986, MNRAS, 221, 1023

Klypin, A., Zhao, H., \& Somerville, R. S. 2002, ApJ, 573, 597

Korchagin, V. I., Girard, T. M., Borkova, T. V., Dinescu, D. I., \& van Altena, W. F. 2003, AJ, 126, 2869

Kroupa, P., Tout, C. A., \& Gilmore, G. 1993, MNRAS, 262, 545

Kuijken, K., \& Gilmore, G. 1991, ApJ, 367, L9

Ludlow, A. D., Navarro, J. F., Springel, V., et al. 2009, ApJ, 692, 931

Maiz-Apellaniz, J. 2001, ApJ, 560, L83

Mashchenko, S., Couchman, H. M. P., \& Wadsley, J. 2006, Nature, 442, 539

Mera, D., Chabrier, G., \& Schaeffer, R. 1998, A\&A, 330, 937

Merrifield, M. R. 1992, AJ, 103, cITA-91-44

Moore, B., Ghigna, S., Governato, F., et al. 1999, ApJ, 524, L19

Naab, T., \& Ostriker, J. P. 2006, MNRAS, 366, 899

Navarro, J. F., Frenk, C. S., \& White, S. D. M. 1997, ApJ, 490, 493

Oh, S.-H., de Blok, W. J. G., Walter, F., Brinks, E., \& Kennicutt, Robert C. J. 2008, AJ, 136, 2761
Ojha, D., Bienayme, O., Robin, A., Creze, M., \& Mohan, V. 1996, A\&A, 311, 456

Olling, R. P., \& Merrifield, M. R. 2001, MNRAS, 326, 164

Purcell, C. W., Bullock, J. S., \& Kaplinghat, M. 2009, ApJ, 703, 2275

Reid, M. J. 2009, Int. J. Mod. Phys., D18, 889

Reid, M. J., \& Brunthaler, A. 2004, ApJ, 616, 872

Reid, M. J., Menten, K. M., Zheng, X. W., et al. 2009, ApJ, 700, 137

Ricotti, M. 2003, MNRAS, 344, 1237

Robin, A. C., Haywood, M., Creze, M., Ojha, D. K., \& Bienayme, O. 1996, A\&A, 305, 125

Sakamoto, T., Chiba, M., \& Beers, T. C. 2003, A\&A, 397, 899

Salucci, P. et al. 2007, MNRAS., 378, 41

Sofue, Y., \& Rubin, V. 2001, ARA\&A, 39, 137

Sofue, Y., Honma, M., \& Omodaka, T. 2008, Astron. Soc. Japan, 61, 227

Sparke, L. S., \& Gallagher, J. S. 2007, Galaxies in the Universe - An Introduction (Cambridge University Press)

Spooner, N. J. 2007, J. Phys. Soc. Jap., 76, 111016

Springel, V., White, S. D. M., Frenk, C. S., et al. 2008a [arXiv:0809.0894]

Springel, W., S. D. M., Frenk, C. S., et al. 2008b, Nature, 456, 73

Strigari, L. E., \& Trotta, R. 2009, JCAP, in press

Wilkinson, M. I., \& Evans, N. W. 1999, MNRAS, 310, 645

Xue, X. X., Rix, H. W., Zhao, G., et al. 2008, ApJ, 684, 1143

Zeilik, M., \& Gregory, S. 1998, Introductory Astronomy and Astrophysics (Saunders College)

Zheng, Z., Flynn, C., Gould, A., Bahcall, J. N., \& Salim, S. 2001, ApJ, 555, 393 